\title{
Maternal Prenatal Somatization, Postnatal Depression, and Offspring Temperament During the COVID-19 Pandemic
}

\author{
Jessica L. Buthmann ${ }^{1}$ \& Ian H. Gotlib ${ }^{1}$ \\ ${ }^{1}$ Department of Psychology, Stanford University \\ 450 Jane Stanford Way, Building 420 \\ Stanford, CA 94305
}

Correspondence:

Jessica L. Buthmann, buthmann@stanford.edu

Word count: 3383

\section{Acknowledgements}

We thank the participants for their contributions. Special thanks to Lucy S. King and Daisy E. Feddoes for their time and effort with data collection and organization.

\section{Financial support}

Funding Statement: This work was supported by the National Institutes of Health (IHG, Grant R37MH101495).

\section{Conflicts of Interest}

Conflicts of interest: None

\section{Ethical Standards}

The authors assert that all procedures contributing to this work comply with the ethical standards of the relevant national and institutional committees on human experimentation and with the Helsinki Declaration of 1975, as revised in 2008. 


\section{Abstract}

Background. Researchers have begun to examine the psychological toll of the ongoing global COVID-19 pandemic. Data are now emerging indicating that there may be long-term adverse effects of the pandemic on new mothers and on children born during this period.

Methods. In a longitudinal study of maternal mental health and child emotional development during the pandemic, we conducted online assessments of a cohort of women at two time points: when they were pregnant at the beginning of the surge of the pandemic in the United States ( $N=725)$, and approximately one year postpartum ( $N=296)$, examining prenatal and postnatal maternal mental health symptoms and infant temperament.

Results. Prenatal maternal symptoms of somatization, the number of people in the household with COVID-19 symptoms, and postnatal maternal depressive symptoms all were positively associated with infant negative affect. Further, postnatal maternal depressive symptoms mediated the relation between prenatal maternal symptoms of somatization and infant negative affect.

Conclusions. These findings have important implications for our understanding of the persistence of mental health symptoms in potentially vulnerable groups and of the emotional development of children who were in utero during the COVID-19 pandemic.

Keywords: COVID-19, somatization, depression, prenatal mental health, infant temperament 
The COVID-19 pandemic has brought devastation on a global scale not seen in over a century. More than five million people have died - more than 740,000 in the United States alone (WHO COVID-19 Dashboard, 2021). The economic turmoil, educational disruption, fear, bereavement, and isolation of the past 18 months have contributed to a marked increase in the prevalence of mental illness. Researchers have now documented increases in symptoms of anxiety, depression, somatization, and posttraumatic stress disorder within the first few months of the global outbreak of the pandemic, especially in women, in people under 40 years of age, in people with a history of mental illness, in students, and in unemployed people (Ran et al., 2020; Xiong et al., 2020). It is critical that we continue to assess the psychological impact of this pandemic in order to minimize its lasting deleterious effects, particularly in vulnerable populations.

Prior to the pandemic, investigators had tested the Developmental Origins of Health and Disease hypothesis (Gluckman et al., 2007), which posits that early experiences, including those occurring during the prenatal period, can lead to a cascade of alterations and adaptations with lifelong consequences for physical and mental health. Findings from these studies indicated that prenatal maternal stress, including natural disasters (Buthmann et al., 2019; Nomura et al., 2019) and maternal difficulties with mental health (Acosta et al., 2019; Davis et al., 2020), is related to poorer emotional development in the offspring.

Researchers have now begun to examine prenatal maternal mental health during the pandemic, as well as factors that predict poorer outcomes in mothers and their children. Most studies in this area have assessed maternal symptoms of depression during the prenatal period. For example, King et al., (2021) found that a sample of women who were pregnant at the beginning of the U.S. surge of the COVID-19 pandemic had higher levels of depressive symptoms than did a matched sample of women who were pregnant several years prior to the pandemic. King et al. found further that symptoms of depression during the pandemic were higher in women who reported greater fear of infection, sociodemographic vulnerability (e.g., 
financial difficulty, being a person of color), and changes to their prenatal care. Other researchers have also reported increases in depressive symptoms during the pandemic in pregnant women who experienced a lack of social support (Filippetti et al., 2021), financial instability (Sherin et al., 2021), and a reduced ability to exercise regularly (Gildner et al., 2020). In addition to depressive symptoms, investigators have also documented elevated levels of stress and anxiety related to the pandemic in pregnant women. Symptoms of anxiety were found to be related to greater fear of infection (Colli et al., 2021) and to changes in prenatal care or in birth plans (Groulx et al., 2021). Feeling unprepared for labor and delivery was associated with medical vulnerability (e.g., chronic illness or having a high-risk pregnancy), changes to prenatal care, and not having access to outdoor space (Preis et al., 2020). In a cross-sectional sample of pregnant women and new mothers, Nomura et al. (2021) found that suicidal ideation and substance use was highest in women who became infected with the virus, and that levels of distress were highest for women in the $3^{\text {rd }}$ trimester and postpartum periods. Finally, fear of the baby contracting COVID-19, lack of social support, and higher levels of pregnancy-specific anxiety have been found to be associated with poorer overall mental health (Pope et al., 2021).

Both depression and anxiety often have comorbid symptoms of somatization, that is, the perception of physical symptoms without an organic cause (De Gucht \& Fischler, 2002; Löwe et al., 2008). It is not surprising, therefore, that somatization symptoms have also risen globally during the pandemic (Jowett et al., 2021; Ran et al., 2020). Few studies, however, have examined somatization symptoms in pregnant women in the context of the pandemic. In China, Xie et al. (2021) found higher symptoms of somatization, depression, anxiety, and hostility in women who were pregnant in 2020 than in women who were pregnant in 2019 , prior to the pandemic. Further, pregnant healthcare workers in China were found to have higher levels of symptoms of somatization, anxiety, and hostility than were non-pregnant female healthcare workers (Liu \& Pérez-Edgar, 2019). 
Although there is a relatively large literature examining the effects of maternal depression on the functioning of offspring (c.f. Gotlib et al., 2020), only a few studies have examined the link between prenatal maternal somatization symptoms and suboptimal child development. For example, prenatal maternal somatization symptoms have been found to be associated with increased cortisone in hair samples in 6-year-old children (Molenaar et al., 2019). In addition, postnatal maternal somatization symptoms have been reported to be related to poorer cognitive skills in young children (Betchen et al., 2020).

Only a small number of researchers have assessed the effects of maternal prenatal mental health during the pandemic on infant functioning. In Italy, Provenzi, et al. (2021a) reported that high levels of prenatal emotional stress and low levels of social support during the pandemic were related to higher symptoms of prenatal maternal anxiety, and that maternal parenting stress and bond with the child mediated the association between prenatal maternal anxiety and reduced regulatory capacity in 3-month-old infants. This research group also found that prenatal maternal stress related to the pandemic was associated with increased SLC6A4 methylation in neonatal buccal samples, which in turn predicted decreased surgency/positive affect in infants at 3 months of age (Provenzi, Mambretti, et al., 2021b). In contrast, Fiske et al. (2021) found that although maternal symptoms of depression increased over the course of the pandemic, there was no relation between this increase in symptoms and infant temperament. The nature of COVID-19 and the related rise in somatization symptoms underscore for the need to examine links between these symptoms in pregnancy and subsequent maternal health and offspring temperament. To examine these associations, we collected data from women who were pregnant at the beginning of the surge of COVID-19 in the United States and followed up with these women at approximately one year postpartum. Given that research examining prenatal and postnatal maternal mental health in the context of the pandemic is in its early stages, the hypotheses we generated are largely exploratory. Nevertheless, we expected that prenatal maternal symptoms of somatization, anxiety, and depression would predict 
postnatal maternal symptoms of depression, and further, that both prenatal and postnatal maternal symptoms would be associated with more dysregulated infant temperament. More specifically, we hypothesized that these symptoms would be associated with the temperament dimension of negative affect, given that high levels of negative emotionality early in life have been associated with risk for the development of psychopathology (Abulizi et al., 2017; De Pauw \& Mervielde, 2010). Finally, we tested whether postnatal depressive symptoms mediated the association between prenatal symptoms and infant temperament.

\section{Methods}

\section{Participants}

We recruited women to participate in the Stanford COVID-19 Perinatal Experiences (COPE) project via online advertisements in April-May of 2020 (T1), as the COVID-19 pandemic surged in the United States (see King et al., 2021 for the full study description). Inclusion criteria into the broader study were that participants be either pregnant or less than 6 months postpartum, and at least 18 years of age. 1,595 women provided informed consent, of whom 1,058 were pregnant. 725 pregnant women completed the survey in full. 516 of these women provided contact information and consented to be contacted in the future for follow up. We followed up at approximately one year postpartum in August-September 2021 (T2) and received responses from 296 participants.

\section{Measures}

Demographic characteristics. At T1, participants reported their age, race, educational attainment, household size, and 2019 household income. At T2, participants reported the date of birth of the child with whom they were pregnant at T1. We calculated an income-to-needs ratio based on household size and income, and the 2019 federal poverty level per household size. See Table 1 for demographic variables of the T1 and T2 samples.

COVID-19 experiences. At both T1 and T2 we administered the COVID-19 and Perinatal Experiences Impact Survey (COPE-IS; Thomason et al., 2020). This survey includes 
questions about medical care, medical history, positive COVID-19 test results, COVID-19specific distress, and COVID-19 symptoms experienced by the participant and members of their household. We summed the number of people in the household who experienced COVID-19 symptoms at T1 to create the variable "household symptoms." COPE-IS asks participants to rate how distressed they were about COVID-19 symptoms or the potential for illness in themselves and in their friends and family on a scale from 0 (mildly distressed) to 6 (extremely distressed). We averaged these two items to create the variable "COVID-19 distress."

Maternal mental health. At T1 we administered the Brief Symptom Inventory (BSI; Derogatis, 1993), an 18-item self-report measure assessing symptoms of depression, anxiety, and somatization. Six items are summed for each subscale; however, because we omitted a question about suicidality, there were only 5 items for the depression scale. Participants rated how often they were distressed by each listed symptom in the past 7 days on a scale from 0 (not at all) to 5 (extremely), yielding a possible range from 0-30 for the anxiety and somatization subscales, and 0-25 for the depression subscale. Cronbach's alphas for the anxiety, somatization, and depression subscales were $.83, .67$, and .85 , respectively, indicating moderate to high reliability within this sample.

We also administered the Edinburgh Postnatal Depression Scale (EPDS; Cox et al., 1987) at T1 and at T2. This 10-item self-report scale asks participants to rate the degree to which statements accurately described their mood in the past 7 days. Participants respond on a scale of 0 (never) to 3 (most of the time), yielding a possible range of $0-30$ for the EPDS. Reliability was high (Cronbach's alpha $=0.85$ at both timepoints). To minimize participant burden, we did not administer the BSI at T2.

Infant temperament. At T2, participants reported on their child's temperament using the Infant Behavior Questionnaire - Very Short Form (IBQ-VSF; Putnam et al., 2014). This 37-item measure asks mothers to indicate the frequency with which their child engaged in specific behaviors in the past 7 days on a scale from 1 (never) to 7 (always). Items are averaged to form 
three subscales: negative affect, surgency/positive affect, and effortful control, with Cronbach's alphas of $.83, .62, .77$, respectively, indicating moderate to high reliability in this sample.

\section{Analysis}

We computed descriptive statistics and correlations among variables of interest using IBM SPSS 27 (Armonk, NY). Based on hypotheses and significant correlations, we used SPSS to conduct a hierarchical linear regression predicting infant negative affect scores. We included demographic variables hypothesized to be related to temperament in step 1 of the regression, including income-to-needs ratio, race, and the child's age. In step 2, we included variables from the prenatal period including maternal somatization symptoms and a sum of the number of people in the family household with COVID-19 symptoms. In the final step we included postnatal maternal depression.

Based on the results of the hierarchical linear regression, we conducted a series of regressions and a mediation analysis in R using the "mediation" package (Tingley et al., 2014). We tested whether postnatal maternal depression symptoms mediated the association between prenatal maternal somatization symptoms and infant negative affect.

\section{Descriptive Statistics}

\section{Results}

Means, standard deviations, and correlations among variables of interest are presented in Table 2. The sample was majority White with a relatively high income-to-needs ratio compared to the federal poverty level. Compared to the full sample of pregnant women at T1, those who participated in the follow up assessment were less likely to be Hispanic/Latinx $(t(720.4)=-3.3, p=.004)$ and more likely to be White $(t(697.8)=-3.3, p<.001)$, to have completed

more years of school $(t(695.6)=-3.1, p=.002)$, to have higher income $(t(722)=-3.0, p=.002)$, and to be older $(t(684.8)=-3.3, p=.001)$; the two groups of women did not differ in scores reflecting prenatal symptoms of somatization, anxiety, or depression (all ps>.44). 
The average age of the children at T2 was approximately 13 months. Negative affect was positively correlated with both surgency and effortful control. A number of variables at T1 and T2 were associated with infant negative affect. Specifically, T1 prenatal maternal somatization symptoms, number of people in the household with COVID-19 symptoms, and income-to-needs ratio predicted infant negative affect at T2, as did T2 postnatal maternal depression symptoms.

\section{Predicting Infant Negative Affect}

We conducted a hierarchical linear regression predicting infant negative affect scores from T1 demographic variables, prenatal maternal somatization, and T2 postnatal depressive symptoms. We examined these variables in three separate steps to determine the proportion of the variance accounted for by each (see Table 3). In step 1, income-to-needs ratio, race, the child's age, and the number of people in the household with COVID-19 symptoms significantly explained $6.1 \%$ of the variation in infant negative affect $(F(4,269)=5.47, p<.001)$. Higher income-to-needs ratio predicted lower infant negative affect scores $(\beta=-.14, p=.018)$, and more people in the household with COVID-19 symptoms during the prenatal period predicted higher infant negative affect scores $(\beta=-.16, p=.007)$.

In step 2 we added prenatal maternal somatization symptoms to the model, which explained an additional $1.6 \%$ of the variance $(F(2,268)=4.80, p=.029)$. Maternal somatization symptoms $(\beta=.13, p=.029)$ predicted higher infant negative affect scores.

In the final step we added postnatal depression symptoms, which explained an additional $1.5 \%$ of the variance in infant negative affect $(\beta=.13, F(1,267)=4.43, p=.036)$. Somatization was no longer significant, but having members of the household with COVID-19 symptoms and income-to-needs ratio were significant, explaining a total of $8.6 \%$ of the variance.

We ran the same regression models predicting surgency and negative affect. Neither model was statistically significant. 


\section{Mediation Model}

Given that somatization symptoms no longer predicted negative affect after accounting for postnatal depression in step 3 of the hierarchical regression, we conducted a series of linear regressions and a formal mediation test to determine whether postnatal maternal depression mediated the association between prenatal maternal somatization and postnatal infant negative affect. Prenatal maternal somatization significantly predicted infant negative affect, controlling for income-to-needs ratio, race, and the age of the child $\left(B=.04, p=.02, R^{2}=.05, F(4,274)=5.02\right.$, $p<.001$, see Figure 1). Prenatal maternal somatization also significantly predicted postnatal maternal depression symptoms $\left(B=.57, p<.001, R^{2}=.13, F(4,269)=11.15, p<.001\right)$. Finally, postnatal maternal depression symptoms were significantly associated with infant negative affect, controlling for income-to-needs ratio, race, and the age of the child $(B=.03, p=.006$, $\left.R^{2}=.06, F(4,269)=5.51, p<.001\right)$.

To conduct a formal test of mediation, we used the "mediation" package for R (Tingley et al., 2014), to compute unstandardized indirect effects for each of 1,000 bootstrapped samples. The bootstrapped unstandardized indirect effect was .01 and the $95 \%$ confidence interval ranged from 0.0004 to $0.03,(p=.048)$, indicating that postnatal depression symptoms mediated the association between prenatal somatization symptoms and infant negative affect.

\section{Discussion}

In this study we examined the relations between prenatal maternal mental health and both postnatal maternal mental health and child temperament during the global COVID-19 pandemic. We found that prenatal maternal symptoms of somatization predicted postnatal maternal depressive symptoms. We found further that negative affect was higher in children of mothers who experienced greater levels of prenatal somatization symptoms, had more people in the household with COVID-19 symptoms, and experienced more postnatal symptoms of depression. Finally, we found that postnatal maternal depression mediated the association 
between prenatal somatization symptoms and infant negative affect, controlling for relevant demographic variables.

This study is among the first longitudinal reports of sustained maternal symptoms of emotional disorder from the prenatal through the postnatal period. López-Morales et al. (2021) found that prenatal maternal symptoms of depression and anxiety increased early in the pandemic following stay-at-home orders in Argentina, and decreased slightly over time.

Longitudinal studies of mental health symptoms in non-pregnant people are beginning to show a decrease in symptoms since the beginning of the pandemic. For example, in the United Kingdom, Fluharty et al. (2021) found that adults reported decreases in their levels of anxiety and depressive symptoms over 21 weeks, especially those who engaged in supportive coping strategies. In a large sample of users of a mental health app, Yarrington et al. (2021) found that symptoms of depression and anxiety both increased at the beginning of the pandemic, but whereas anxiety symptoms returned to baseline levels within months, depressive symptoms continued to increase during the pandemic. More research is needed to determine the persistence of mental health symptoms and to identify which groups are most susceptible to lasting effects. In this context, pregnant women, new mothers, and other vulnerable groups may experience prolonged symptoms of emotional disturbance, even as social isolation decreases and COVID-19 vaccines become widely available in the United States.

Our results are largely consistent with findings reported prior to the pandemic of associations between poor prenatal maternal mental health, whether defined as stress (e.g., Buthmann et al., 2019), depression (e.g., Borchers et al., 2021), or anxiety (e.g., Van den Bergh et al., 2008), and subsequent difficulties in offsprings' emotional development. Fewer studies have examined maternal somatization in relation to offspring functioning. Nevertheless, given the increase in somatization documented during the pandemic (Jowett et al., 2021; Ran et al., 2020), we hypothesized that maternal somatization would affect infant temperament. Although we did not collect biological specimens in our study to elucidate mechanisms that might underlie 
the relations between maternal mental health and infant temperament, prior research testing the Developmental Origins of Health and Disease hypothesis (Gluckman et al., 2007) implicates epigenetic, neuroendocrine, and/or inflammatory pathways in this association. Future research is required to determine whether elevated negative affect in infants of mothers who experienced high symptoms of somatization during the pandemic will be sustained or normalize over time, and whether levels of negative emotionality in these infants predict the subsequent development of psychopathology, as has been documented by other investigators (Abulizi et al., 2017; Liu \& Pérez-Edgar, 2019).

We expected that prenatal maternal symptoms of anxiety and depression early in the pandemic would predict infant negative affect as well as maternal somatization symptoms. Although prenatal depressive and anxious symptoms did not predict subsequent functioning, it is important to note that symptoms of somatization were positively correlated with concurrent symptoms of anxiety and depression in the prenatal period. Somatization scores might have reflected distress about contracting COVID-19 and had a lasting impact on maternal mental health (i.e., postnatal depression) in a way that levels of anxiety and depression did not. Notably, participants' ratings of distress about COVID-19 symptoms and illness in themselves and friends and family were associated with increased symptoms of somatization. Consistent with Motivated Cue-Integration theory (Shalev, 2021) in the context of COVID-19, a lack of certainty about the facts of the disease in the spring of 2020 , especially concerning its effects on pregnancy, known variability in symptom presentation, and a heightened sense of threat (Jowett et al., 2021) may have contributed to hypervigilant monitoring of physical symptoms.

We should note three limitations of this study. First, our sample was composed primarily of White people of high socioeconomic status; thus, the generalizability of our findings to other populations is not clear. Second, data were self-reported via online survey, and it is possible that there was bias in participants' responses. This is particularly important for the reporting of infant temperament, although recent reports have found little evidence of systematic bias as a 
function of parental psychopathology (Olino et al., 2020). Finally, although we demonstrated that postnatal maternal depressive symptoms mediated the association between prenatal maternal somatization symptoms and infant negative affect, we did not assess postnatal somatization symptoms and, therefore, cannot determine whether somatization symptoms in the postnatal period would have been a stronger predictor of infant negative affect than were postnatal depressive symptoms.

Despite these limitations, we document in this study that prenatal maternal symptoms of somatization at the beginning of the COVID-19 surge in the United States predict postnatal maternal symptoms of depression, which in turn mediate the association between prenatal somatization and infant negative affect. Further research is needed to examine trajectories of mental health symptoms of mothers who gave birth during the pandemic and associations with trajectories of offspring emotional development. Elucidating factors that affect risk and resilience in children in utero during the COVID-19 pandemic will help public health officials and healthcare providers to identify groups in need of more resources or support in order to mitigate the psychological toll of the pandemic. 


\section{References}

Abulizi, X., Pryor, L., Michel, G., Melchior, M., Waerden, J. van der, \& Group, on behalf of T. E. M.-C. C. S. (2017). Temperament in infancy and behavioral and emotional problems at age 5.5: The EDEN mother-child cohort. PLOS ONE, 12(2), e0171971. https://doi.org/10.1371/journal.pone.0171971

Acosta, H., Tuulari, J. J., Scheinin, N. M., Hashempour, N., Rajasilta, O., Lavonius, T. I., Pelto, J., Saunavaara, V., Parkkola, R., Lähdesmäki, T., Karlsson, L., \& Karlsson, H. (2019). Maternal pregnancy-related anxiety is associated with sexually dimorphic alterations in amygdala volume in 4-year-old children. Frontiers in Behavioral Neuroscience, 13. https://doi.org/10.3389/fnbeh.2019.00175

Betchen, M., Grunberg, V. A., Gringlas, M., \& Cardonick, E. (2020). Being a mother after a cancer diagnosis during pregnancy: Maternal psychosocial functioning and child cognitive development and behavior. Psycho-Oncology, 29(7), 1148-1155. https://doi.org/10.1002/pon.5390

Borchers, L. R., Dennis, E. L., King, L. S., Humphreys, K. L., \& Gotlib, I. H. (2021). Prenatal and postnatal depressive symptoms, infant white matter, and toddler behavioral problems. Journal of Affective Disorders, 282, 465-471. https://doi.org/10.1016/j.jad.2020.12.075

Buthmann, J., Finik, J., Ventura, G., Zhang, W., Shereen, A. D., \& Nomura, Y. (2019). The children of Superstorm Sandy: Maternal prenatal depression blunts offspring electrodermal activity. Biological Psychology, 146, 107716. https://doi.org/10.1016/j.biopsycho.2019.107716

Colli, C., Penengo, C., Garzitto, M., Driul, L., Sala, A., Degano, M., Preis, H., Lobel, M., \& Balestrieri, M. (2021). Prenatal stress and psychiatric symptoms during early phases of the COVID-19 pandemic in Italy. International Journal of Women's Health, 13, 653-662. https://doi.org/10.2147/IJWH.S315467 
Davis, E. P., Hankin, B. L., Glynn, L. M., Head, K., Kim, D. J., \& Sandman, C. A. (2020).

Prenatal maternal stress, child cortical thickness, and adolescent depressive symptoms. Child Development, 91(2), e432-e450. https://doi.org/10.1111/cdev.13252

De Gucht, V., \& Fischler, B. (2002). Somatization: A critical review of conceptual and methodological issues. Psychosomatics, 43(1), 1-9. https://doi.org/10.1176/appi.psy.43.1.1

De Pauw, S. S. W., \& Mervielde, I. (2010). Temperament, personality and developmental psychopathology: A review based on the conceptual dimensions underlying childhood traits. Child Psychiatry \& Human Development, 41(3), 313-329. https://doi.org/10.1007/s10578-009-0171-8

Derogatis, L. R. (1993). BSI Brief Symptom Inventory. Administration, Scoring, and Procedures Manual. https://ci.nii.ac.jp/naid/20000821855/

Filippetti, M. L., Clarke, A., \& Rigato, S. (2021). The mental health crisis of expectant women in the UK: Effects of the COVID-19 pandemic on prenatal mental health, antenatal attachment and social support. PsyArXiv. https://doi.org/10.31234/osf.io/4328p

Fiske, A., Scerif, G., \& Holmboe, K. (2021). Maternal depressive symptoms and early childhood temperament before and during the COVID-19 pandemic in the United Kingdom. PsyArXiv. https://doi.org/10.31234/osf.io/quket

Fluharty, M., Bu, F., Steptoe, A., \& Fancourt, D. (2021). Coping strategies and mental health trajectories during the first 21 weeks of COVID-19 lockdown in the United Kingdom. Social Science \& Medicine, 279, 113958. https://doi.org/10.1016/j.socscimed.2021.113958

Gildner, T. E., Laugier, E. J., \& Thayer, Z. M. (2020). Exercise routine change is associated with prenatal depression scores during the COVID-19 pandemic among pregnant women across the United States. PLOS ONE, 15(12), e0243188. https://doi.org/10.1371/journal.pone.0243188 
Gluckman, P. D., Hanson, M. A., \& Beedle, A. S. (2007). Early life events and their consequences for later disease: A life history and evolutionary perspective. American Journal of Human Biology, 19(1), 1-19. https://doi.org/10.1002/ajhb.20590

Gotlib, I. H., Goodman, S. H., \& Humphreys, K. L. (2020). Studying the intergenerational transmission of risk for depression: Current status and future directions. Current Directions in Psychological Science, 29(2), 174-179. https://doi.org/10.1177/0963721420901590

Groulx, T., Bagshawe, M., Giesbrecht, G., Tomfohr-Madsen, L., Hetherington, E., \& Lebel, C. A. (2021). Prenatal care disruptions and associations with maternal mental health during the COVID-19 pandemic. Frontiers in Global Women's Health, 2, 20. https://doi.org/10.3389/fgwh.2021.648428

Jowett, S., Shevlin, M., Hyland, P., \& Karatzias, T. (2021). Posttraumatic stress disorder and persistent somatic symptoms during the COVID-19 Pandemic: The role of sense of threat. Psychosomatic Medicine, 83(4), 338-344. https://doi.org/10.1097/PSY.0000000000000890

King, L. S., Feddoes, D. E., Kirshenbaum, J. S., Humphreys, K. L., \& Gotlib, I. H. (2021). Pregnancy during the pandemic: The impact of COVID-19-related stress on risk for prenatal depression. Psychological Medicine, 1-11. https://doi.org/10.1017/S003329172100132X

Liu, P., \& Pérez-Edgar, K. E. (2019). Developmental pathways from early behavioral inhibition to later anxiety: an integrative review of developmental psychopathology research and translational implications. Adolescent Research Review, 4(1), 45-58. https://doi.org/10.1007/s40894-018-0092-5

López-Morales, H., del-Valle, M. V., Andrés, M. L., Gelpi Trudo, R., Canet-Juric, L., \& Urquijo, S. (2021). Longitudinal study on prenatal depression and anxiety during the COVID-19 
pandemic. Archives of Women's Mental Health. https://doi.org/10.1007/s00737-021$01152-1$

Löwe, B., Spitzer, R. L., Williams, J. B. W., Mussell, M., Schellberg, D., \& Kroenke, K. (2008). Depression, anxiety and somatization in primary care: Syndrome overlap and functional impairment. General Hospital Psychiatry, 30(3), 191-199. https://doi.org/10.1016/j.genhosppsych.2008.01.001

Molenaar, N. M., Tiemeier, H., van Rossum, E. F. C., Hillegers, M. H. J., Bockting, C. L. H., Hoogendijk, W. J. G., van den Akker, E. L., Lambregtse-van den Berg, M. P., \& El Marroun, H. (2019). Prenatal maternal psychopathology and stress and offspring HPA axis function at 6 years. Psychoneuroendocrinology, 99, 120-127. https://doi.org/10.1016/j.psyneuen.2018.09.003

Nomura, Y., Davey, K., Pehme, P., Finik, J., Glover, V., Zhang, W., Huang, Y., Buthmann, J., Dana, K., Yoshida, S., Tsuchiya, K., Li, X., \& Ham, J. (2019). Influence of in-utero exposure to maternal depression and natural disaster-related stress on infant temperament at 6 months: The children of Superstorm Sandy. Infant Mental Health Journal, 4O(2), 204-216. https://doi.org/DOI: 10.1002/imhj.21766

Nomura, Y., Kittler, P., Taveras, S., Sie, S. Y., Nelson, E., Davey, K., Kaushal, R., Rajendran, K., Gordon, A., Shereen, A. Duke., \& Phan, H. (2021). Psychological effects of COVID19 on pregnant women and new mothers living in a US hotspot [Preprint]. In Review. https://doi.org/10.21203/rs.3.rs-669031/v1

Olino, T. M., Guerra-Guzman, K., Hayden, E. P., \& Klein, D. N. (2020). Evaluating maternal psychopathology biases in reports of child temperament: An investigation of measurement invariance. Psychological Assessment, 32(11), 1037-1046. https://doi.org/10.1037/pas0000945 
Pope, J., Olander, E. K., Leitao, S., Meaney, S., \& Matvienko-Sikar, K. (2021). Prenatal stress, health, and health behaviours during the COVID-19 pandemic: An international survey. Women and Birth. https://doi.org/10.1016/j.wombi.2021.03.007

Preis, H., Mahaffey, B., Heiselman, C., \& Lobel, M. (2020). Vulnerability and resilience to pandemic-related stress among U.S. women pregnant at the start of the COVID-19 pandemic. Social Science \& Medicine, 266, 113348. https://doi.org/10.1016/j.socscimed.2020.113348

Provenzi, L., Grumi, S., Altieri, L., Bensi, G., Bertazzoli, E., Biasucci, G., Cavallini, A., Decembrino, L., Falcone, R., Freddi, A., Gardella, B., Giacchero, R., Giorda, R., Grossi, E., Guerini, P., Magnani, M. L., Martelli, P., Motta, M., Nacinovich, R., ... Group, M.-C. S. (2021a). Prenatal maternal stress during the COVID-19 pandemic and infant regulatory capacity at 3 months: A longitudinal study. Development and Psychopathology, 1-9. https://doi.org/10.1017/S0954579421000766

Provenzi, L., Mambretti, F., Villa, M., Grumi, S., Citterio, A., Bertazzoli, E., Biasucci, G., Decembrino, L., Falcone, R., Gardella, B., Longo, R., Nacinovich, R., Pisoni, C., Prefumo, F., Orcesi, S., Scelsa, B., Giorda, R., \& Borgatti, R. (2021b). Hidden pandemic: COVID-19-related stress, SLC6A4 methylation and infants' temperament at 3 months [Preprint]. In Review. https://doi.org/10.21203/rs.3.rs-492196/v1

Putnam, S. P., Helbig, A. L., Gartstein, M. A., Rothbart, M. K., \& Leerkes, E. (2014). Development and assessment of short and very short forms of the Infant Behavior Questionnaire-Revised. Journal of Personality Assessment, 96(4), 445-458. https://doi.org/10.1080/00223891.2013.841171

Ran, L., Wang, W., Ai, M., Kong, Y., Chen, J., \& Kuang, L. (2020). Psychological resilience, depression, anxiety, and somatization symptoms in response to COVID-19: A study of the general population in China at the peak of its epidemic. Social Science \& Medicine, 262, 113261. https://doi.org/10.1016/j.socscimed.2020.113261 
Shalev, I. (2021). Theory of motivated cue-integration and COVID-19: Between interoception, somatization, and radicalization. Frontiers in Psychiatry, 8, 631758. https://doi.org/10.3389/fpsyt.2021.631758

Sherin, M., Gildner, T. E., \& Thayer, Z. M. (2021). COVID-19-related changes to pregnant people's work-plans increase prenatal depression. Frontiers in Global Women's Health, 2, 19. https://doi.org/10.3389/fgwh.2021.639429

Thomason, M. E., Graham, A., \& Vantieghem, M. R. (2020). COPE: Coronavirus Perinatal Experiences-Impact Survey (COPE-IS). osf. io/uqhcv

Tingley, D., Yamamoto, T., Hirose, K., Keele, L., \& Imai, K. (2014). mediation: R package for causal mediation analysis. UCLA Statistics/American Statistical Association. https://dspace.mit.edu/handle/1721.1/91154

Van den Bergh, B. R. H., Van Calster, B., Smits, T., Van Huffel, S., \& Lagae, L. (2008). Antenatal maternal anxiety is related to HPA-axis dysregulation and self-reported depressive symptoms in adolescence: A prospective study on the fetal origins of depressed mood. Neuropsychopharmacology, 33(3), 536-545.

https://doi.org/10.1038/sj.npp.1301450

WHO COVID-19 Dashboard. (2021). https://covid19.who.int

Xie, M., Wang, X., Zhang, J., \& Wang, Y. (2021). Alteration in the psychologic status and family environment of pregnant women before and during the COVID-19 pandemic. International Journal of Gynecology \& Obstetrics, 153(1), 71-75. https://doi.org/10.1002/ijgo.13575

Xiong, J., Lipsitz, O., Nasri, F., Lui, L. M. W., Gill, H., Phan, L., Chen-Li, D., Iacobucci, M., Ho, R., Majeed, A., \& Mclntyre, R. S. (2020). Impact of COVID-19 pandemic on mental health in the general population: A systematic review. Journal of Affective Disorders, 277, 55-64. https://doi.org/10.1016/j.jad.2020.08.001 
Yarrington, J. S., Lasser, J., Garcia, D., Vargas, J. H., Couto, D. D., Marafon, T., Craske, M. G., \& Niles, A. N. (2021). Impact of the COVID-19 Pandemic on Mental Health among 157,213 Americans. Journal of Affective Disorders, 286, 64-70.

https://doi.org/10.1016/j.jad.2021.02.056 
Table 1. Demographic characteristics of the sample at T1 and T2.

\begin{tabular}{lcl}
\hline & $\mathrm{T} 1(\mathrm{n}=725)$ & $\mathrm{T} 2(\mathrm{n}=296)$ \\
\hline Race & & \\
White & $67.4 \%$ & $69.6 \%$ \\
Asian/Asian American & $18.3 \%$ & $14.2 \%$ \\
Black/African American & $1.4 \%$ & $0.7 \%$ \\
Hispanic/Latinx & $14.5 \%$ & $5.7 \%$ \\
Native American & $1.0 \%$ & $0.3 \%$ \\
Pacific Islander & $1.4 \%$ & $1.0 \%$ \\
Other & $2.3 \%$ & $1.0 \%$ \\
Maternal age (T1) & $19-50(\mathrm{M}=33.7, \mathrm{SD}=4.4)$ & $23-50(\mathrm{M}=34.3, \mathrm{SD}=4.0)$ \\
Maternal educational attainment (T1) & $84 \%$ & $92 \%$ \\
(\% college degree+) & & \\
\hline
\end{tabular}


Table 2. Means, standard deviations, and correlations among variables of interest

\begin{tabular}{|c|c|c|c|c|c|c|c|c|c|c|c|c|c|}
\hline & $\mathrm{M}(\mathrm{SD})$ & 1. & 2. & 3. & 4. & 5. & 6. & 7. & 8. & 9. & 10. & 11. & 12. \\
\hline 1. Negative affect & $4.4(1.0)$ & - & & & & & & & & & & & \\
\hline 2. Effortful control & $5.4(0.7)$ & $-.11^{\dagger}$ & - & & & & & & & & & & \\
\hline 3. Surgency & $5.5(0.6)$ & $.15^{*}$ & $.43^{* *}$ & - & & & & & & & & & \\
\hline 4. Child age & $13.4(2.0)$ & .06 & .08 & -.01 & - & & & & & & & & \\
\hline $\begin{array}{l}\text { 5. Income-to- } \\
\text { needs }\end{array}$ & $9.0(4.0)$ & $-.19^{* *}$ & -.04 & .01 & -.10 & - & & & & & & & \\
\hline $\begin{array}{l}\text { 6. Household } \\
\text { Symptoms }\end{array}$ & $0.3(0.8)$ & $.18^{\star *}$ & -.02 & -.05 & -.03 & $-.20^{* *}$ & - & & & & & & \\
\hline $\begin{array}{l}\text { 7. COVID-19 } \\
\text { Distress }\end{array}$ & $2.5(1.7)$ & .09 & .08 & .01 & .05 & -.09 & $.13^{*}$ & - & & & & & \\
\hline $\begin{array}{l}\text { 8. BSI } \\
\text { Somatization }\end{array}$ & $3.0(3.2)$ & $.18^{* *}$ & -.03 & -.07 & .02 & $-.19^{* *}$ & .05 & $.18^{* *}$ & - & & & & \\
\hline 9. BSI Anxiety & $4.7(4.5)$ & .09 & .08 & .05 & .08 & $-.11^{\dagger}$ & .02 & $.31^{* *}$ & $.57^{* *}$ & - & & & \\
\hline $\begin{array}{l}\text { 10. BSI } \\
\text { Depression }\end{array}$ & $4.4(3.9)$ & .09 & -.02 & -.02 & .03 & -.09 & -.01 & $.31^{* *}$ & $.50^{* *}$ & $.74^{\star *}$ & - & & \\
\hline $\begin{array}{l}\text { 11. Prenatal } \\
\text { EPDS }\end{array}$ & $9.4(5.0)$ & .04 & -.05 & -.05 & -.01 & -.04 & .04 & $.18^{* *}$ & $.44^{* *}$ & $.71^{* *}$ & $.72^{* *}$ & - & \\
\hline $\begin{array}{l}\text { 11. Postnatal } \\
\text { EPDS }\end{array}$ & $7.7(5.1)$ & $.18^{* *}$ & $-.11^{\dagger}$ & $-.13^{*}$ & .05 & -.08 & .02 & $.18^{* *}$ & $.37^{\star *}$ & $.43^{* *}$ & $.46^{\star *}$ & $.58^{* *}$ & - \\
\hline
\end{tabular}

Note: ${ }^{\dagger} p<.1,{ }^{*} p<.05,{ }^{* *} p<.01$

Income-to-needs=the household income divided by the federal poverty level for the household size. Household Symptoms=number of people in the household with COVID=19 symptoms during the prenatal period

$\mathrm{BSI}=$ Brief Symptom Inventory (all from the prenatal period)

EPDS=Edinburgh Postnatal Depression Scale 
Table 3. Hierarchical regression infant predicting negative affect scores

\begin{tabular}{|c|c|c|c|c|c|c|c|c|}
\hline & \multicolumn{8}{|c|}{$95 \% \mathrm{Cl}$} \\
\hline & $B$ & $S E B$ & $\beta$ & Lower & Upper & Adjusted $R^{2}$ & $\Delta R^{2}$ & $p$ \\
\hline Step 1 & & & & & & .061 & .075 & $<.001$ \\
\hline Income-needs & -.043 & .015 & $-.144^{\star}$ & -.071 & -.014 & & & \\
\hline Race & .055 & .031 & .104 & -.007 & .117 & & & \\
\hline Child age & .022 & .029 & .055 & -.034 & .079 & & & \\
\hline $\begin{array}{l}\text { Household } \\
\text { Symptoms }\end{array}$ & .205 & .076 & $.161^{\star *}$ & .055 & .355 & & & \\
\hline Step 2 & & & & & & .075 & .016 & .029 \\
\hline Income-needs & -.029 & .015 & $-.121^{\star}$ & -.058 & .000 & & & \\
\hline Race & .051 & .031 & .098 & -.009 & .112 & & & \\
\hline Child age & .026 & .028 & .054 & -.029 & .081 & & & \\
\hline $\begin{array}{l}\text { Household } \\
\text { Symptoms }\end{array}$ & .205 & .076 & $.161^{* *}$ & .056 & .354 & & & \\
\hline Somatization & .039 & .018 & $.130^{\star}$ & .004 & .074 & & & \\
\hline Step 3 & & & & & & .086 & .015 & .036 \\
\hline Income-needs & -.029 & .015 & $-.122^{\star}$ & -.058 & .000 & & & \\
\hline Race & .048 & .031 & .091 & -.013 & .108 & & & \\
\hline Child age & .023 & .028 & .048 & -.032 & .078 & & & \\
\hline $\begin{array}{l}\text { Household } \\
\text { symptoms }\end{array}$ & .205 & .075 & $.161^{* *}$ & .057 & .353 & & & \\
\hline Somatization & .025 & .019 & .082 & -.013 & .062 & & & \\
\hline $\begin{array}{l}\text { Postnatal } \\
\text { depression }\end{array}$ & .025 & .012 & $.131^{*}$ & .002 & .049 & & & \\
\hline
\end{tabular}

Note: ${ }^{*} \mathrm{p} \leq .05,{ }^{* *} \mathrm{p}<.01$

Income-to-needs=the household income divided by the federal poverty level for the household size. Household Symptoms=number of people in the household with COVID=19 symptoms during the prenatal period. 
Figure 1. Postnatal maternal depressive symptoms mediate the association between prenatal maternal somatization symptoms and infant negative affect

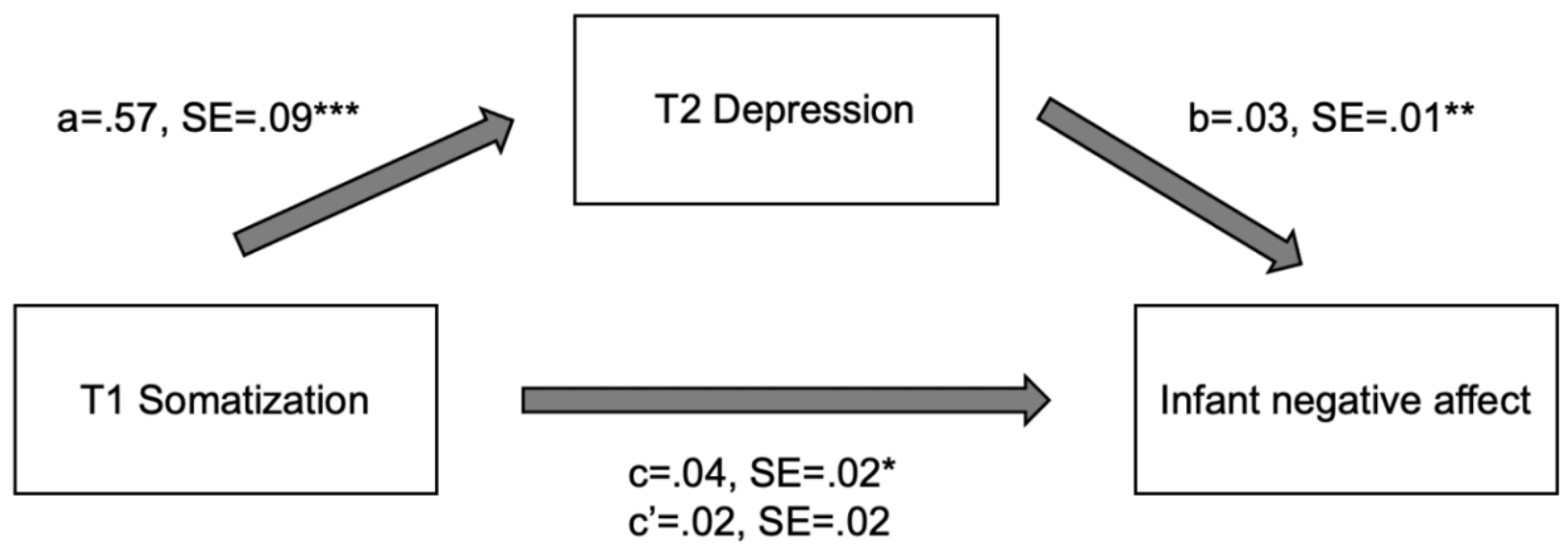

Note: ${ }^{*} p<.05,{ }^{* *} p<.01,{ }^{* *} p<.001 . T 1$ refers to the prenatal period. T2 refers to the postnatal period. Paths $a, b, c$, and c' represent unstandardized regression coefficients. Path c refers to the total effect of prenatal somatization symptoms on infant negative affect. Path c' refers to the direct effect. $N=274$. 